\title{
Algebraic cobordisms of a Pfister quadric
}

\author{
A.Vishik* , N.Yagita
}

July 4, 2005

\section{Introduction}

The aim of this paper is to compute algebraic cobordisms of Pfister quadric $Q_{\alpha}$. We present two different approaches to this problem. The first one uses the motivic spectrum $M G L$ (constructed by V.Voevodsky in [19]), and is reduced to the computation of $M G L^{2 * * *}\left(Q_{\alpha}\right)$, and the second one uses the definition of algebraic cobordism $\Omega^{*}\left(Q_{\alpha}\right)$ due to M.Levine and F.Morel (see $[4],[5],[6])$. Consequently, the methods of computations are quite different. The more pleasant is the fact that the results do agree, especially, since, in general, it is not proven that $M G L^{2 i, i}(X)=\Omega^{i}(X)$ (of course, it hardly could be other way). We hope, that our two independent computations will enable the reader to see the problem from different angles. The case of a Pfister quadric is one of the few noncellular varieties for which algebraic cobordism ring is completely computed (the others are $B \mathbb{Z} / p$ and certain reductive groups - see [23]).

Certainly, the fact that $Q_{\alpha}$ is geometrically cellular helped here a lot. But another ingredient which permitted such computation to happen is the fact that the change of constants map to the algebraic closure appears to be injective in our case. In general (even for geometrically cellular varieties), it is not so. Nevertheless, one could expect that the same could happen for certain classes of varieties, say, for generically cellular ones (that is, ones which become cellular over own generic point). The computation of algebraic cobordisms for such varieties would be the next natural task.

*supported by the Weyl fund and by RFBR grant 22005 
We would like to thank F.Morel for suggesting that the statement like Corollary 2.8 should be true, and A.Nenashev and K.Zainoullin for many questions and remarks which substantially improved the exposition in Section 2. Also, we are very grateful to B.Kahn, I.Panin, A.Smirnov and B.Totaro for very useful discussions. This text was written while the first author was a member of the Institute for Advanced Study at Princeton and the Institut des Hautes Études Scientifiques, and he would like to express his gratitude to both institutions for the support and excellent working conditions.

\section{Cobordism motives}

Everywhere in this text we assume that the base field $k$ has characteristic 0 . Let $A^{*}(\mathrm{X})$ be any oriented (generalised) cohomology theory on the category of smooth algebraic varieties over the field $k$ (see [5]).

An oriented cohomology theory is a contravariant functor from the category of smooth varieties (over $k$ ) to the category of graded rings which is equipped with the structure of push-forward maps for projective equidimensional morphisms (with appropriate shift in the grading) satisfying the axioms (A1)-(A4) in [5]. The algebraic cobordism theory $\Omega^{*}(X)($ see $[5,6,4])$ is defined as the universal theory among the oriented cohomology theories.

Following [8] we define the category of $A$-motives denoted $\mathcal{M}_{A}$ as follows. We start with the category of $A$-correspondences (of degree 0) $\operatorname{Cor}_{A}^{0}$ whose objects are the classes $[X]$ of smooth projective varieties over $k$, and $\operatorname{Mor}_{C_{\text {or }}{ }_{A}^{0}}([X],[Y])=A^{\operatorname{dim}(Y)}(X \times Y)$. The composition of morphisms is defined in a usual way: $\varphi \circ \psi:=p_{X \times Z *}\left(p_{X \times Y}^{*}(\varphi) \cdot p_{Y \times Z}^{*}(\psi)\right)$, where $p_{X \times Y}, p_{Y \times Z}$ and $p_{X \times Z}$ are projection from $X \times Y \times Z$ onto $X \times Y, Y \times Z$ and $X \times Z$, respectively. Then, $\mathcal{M}_{A}$ is a Karoubian envelope of $\operatorname{Cor}_{A}^{0}$. It is naturally a tensor additive category. Typical object of $\mathcal{M}_{A}$ is the pair $([X], p)$, where $X$ is smooth projective variety over $k$ and $p \in \operatorname{End}_{C_{\text {or }}^{0}}([X])$ is some projector $(p \circ p=p)$. Hence, for each $M \in \mathcal{M}_{A}$, we can define the $A^{*}(\operatorname{Spec}(k))$-module $A^{*}(M)$

In the case of a Chow groups, $\mathcal{M}_{C H}$ is nothing else, but the well-known category of effective Chow-motives $C_{\text {how }}{ }^{e f f}(k)$. Consider now the case when $A^{*}(X)$ is the algebraic cobordism theory $\Omega^{*}(X)$ constructed by M.Levine and F.Morel (see $[5,6,4]$ ), or $M G L^{2 * * *}(X)$ constructed by V.Voevodsky. In both cases we have natural morphism $\operatorname{fog}_{A}: A^{*}(X) \rightarrow C H^{*}(X)$ of generalized 
cohomology theories. It induces functor $\mathcal{M}_{A} \stackrel{\text { fog }}{\rightarrow} \mathcal{M}_{C H}$.

The results about $\mathcal{M}_{\Omega}$ or $\mathcal{M}_{M G L}$ we need will follow from some general statements about additive categories. So, we pass to those.

We say that we are in the situation $(\star)$, if we have an additive functor $F: \mathcal{C} \rightarrow \mathcal{D}$ between the additive categories such that the following three conditions are satisfied:

0) $F$ is surjective on the isomorphism classes of objects;

1) $F$ is surjective on morphisms;

2) For any $X \in O b(\mathcal{C})$, the kernel of the ring homomorphism $F_{X}: \operatorname{End}_{\mathcal{C}}(X) \rightarrow \operatorname{End}_{\mathcal{D}}(F(X))$ consists of nilpotents.

Lemma 2.1 Let us be in the situation $(\star)$. Suppose $\alpha \in H_{\mathcal{C}}(X, Y)$ be such morphism that $F(\alpha)$ is an isomorphism. Then so is $\alpha$.

Proof: Since $F$ is surjective on morphisms, there exists $\beta \in \operatorname{Hom}_{\mathcal{C}}(Y, X)$ such that $F(\beta) \circ F(\alpha)=1_{F(X)}$ and $F(\alpha) \circ F(\beta)=1_{F(Y)}$. Then $\beta \circ \alpha=1_{X}+x$ and $\alpha \circ \beta=1_{Y}+y$, where $x$ and $y$ belong to the kernel of $F_{X}$ and $F_{Y}$, respectively, and so, are nilpotent.

Use induction on the maximum of nilpotence exponents of $x$ and $y$. If such exponent is 1 , we are done. Otherwise, consider $\beta^{\prime}:=\beta \circ\left(1_{Y}-y\right)$. Notice, that $\alpha \circ x=y \circ \alpha$ and $x \circ \beta=\beta \circ y$. Then $\alpha \circ \beta^{\prime}=\left(1_{Y}+y\right) \circ\left(1_{Y}-y\right)=1_{Y}-y^{2}$ and $\beta^{\prime} \circ \alpha=\beta \circ \alpha-\beta \circ y \circ \alpha=1_{X}+x-x \circ\left(1_{X}+x\right)=1_{X}-x^{2}$. The maximum of nilpotence exponents of $-x^{2}$ and $-y^{2}$ is smaller than that of $x$ and $y$ (since the latter was greater than 1). By induction, we are done.

As a corollary, we immediately get:

Proposition 2.2 Let us be in the situation $(\star)$. Then the functor $F$ defines a 1-to-1 correspondence $I s o(\mathcal{C}) \rightarrow I s o(\mathcal{D})$ between the set of isomorphism classes of objects of $\mathcal{C}$ and $\mathcal{D}$.

Proof: By condition 0), our map $I s o(\mathcal{C}) \rightarrow I s o(\mathcal{D})$ is surjective, and by Lemma 2.1 and condition 1), it is injective. 
Proposition 2.3 Suppose we are in the situation $(\star)$. Then $F$ is surjective on projectors. That is, for any $X \in O b(\mathcal{C})$ and any projector $p_{F(X)} \in$ $\operatorname{End}_{\mathcal{D}}(F(X))$ there exists projector $p_{X} \in \operatorname{End}_{\mathcal{C}}(X)$ such that $F\left(p_{X}\right)=p_{F(X)}$.

Proof: Since $F$ is surjective on morphisms, there exists $p \in \operatorname{End}_{\mathcal{C}}(X)$ such that $F(p)=p_{F(X)}$.

Consider polynomials $\phi_{n}(t), n \geqslant 1$ defined inductively as follows: $\phi_{1}(t)=$ $\left(2 t-t^{2}\right)^{2}, \phi_{n+1}(t)=\phi_{1}\left(\phi_{n}(t)\right)$. Now, it is sufficient to apply the following lemma to the homomorphism $F_{X}: \operatorname{End}_{\mathcal{C}}(X) \rightarrow \operatorname{End}_{\mathcal{D}}(F(X))$.

Lemma 2.4 Let $\pi: A \rightarrow B$ be a homomorphism of associative rings, such that $\operatorname{ker}(\pi)$ consists of nilpotents. Let $a \in A, b \in B$ be such that $\pi(a)=b$ and $b$ is an idempotent. Then for sufficiently large $n, \phi_{n}(a)$ is an idempotent, and $\pi\left(\phi_{n}(a)\right)=b$.

Proof: Really, for arbitrary $c \in A$ consider $x(c):=c^{2}-c$. Then $x(a)$ is nilpotent ( $b$ is idempotent). But $x\left(\phi_{1}(c)\right)$ is divisible by $x(c)^{2}$ (in the commutative subring of $A$ generated by $c)$. Thus, $x\left(\phi_{n}(a)\right)$ is divisible by $x(a)^{2^{n}}$, and so, is zero, for large $n$.

Notice, that, after all, $p_{X}$ can be expressed as a polynomial of $p$ (with $\mathbb{Z}$-coefficients) without the constant term.

In general, such lifting $p_{X}$ is not unique. Nevertheless, the different liftings give isomorphic objects of the Karoubian envelope of $\mathcal{C}$.

Consider Karoubian envelopes $\mathcal{P} \mathcal{A}(\mathcal{C})$ and $\mathcal{P} \mathcal{A}(\mathcal{D})$ of the categories $\mathcal{C}$ and $\mathcal{D}$. We have natural functor $\mathcal{P} \mathcal{A}(F): \mathcal{P} \mathcal{A}(\mathcal{C}) \rightarrow \mathcal{P} \mathcal{A}(\mathcal{D})$.

Proposition 2.5 Suppose the functor $F: \mathcal{C} \rightarrow \mathcal{D}$ satisfies the conditions $(\star)$. Then so does the functor $\mathcal{P} \mathcal{A}(F): \mathcal{P} \mathcal{A}(\mathcal{C}) \rightarrow \mathcal{P} \mathcal{A}(\mathcal{D})$.

Proof: Condition 0): Any object of $\mathcal{P} \mathcal{A}(\mathcal{D})$ has the form $\left(U, p_{U}\right)$, where $U \in O b(\mathcal{D})$ and $p_{U} \in \operatorname{End}_{\mathcal{D}}(U)$ is a projector. By condition 0$)$, there exists $X \in O b(\mathcal{C})$ such that $U$ is isomorphic to $F(X)$. Then there exists projector $p_{F(X)} \in \operatorname{End}_{\mathcal{D}}(F(X))$ such that $\left(F(X), p_{F(X)}\right)$ is isomorphic to $\left(U, p_{U}\right)$ in $\mathcal{P} \mathcal{A}(\mathcal{D})$. By Proposition 2.3, there exists projector $p_{X} \in \operatorname{End}_{\mathcal{C}}(X)$ such that $\left(F(X), p_{F(X)}\right)=\mathcal{P} \mathcal{A}(F)\left(\left(X, p_{X}\right)\right)$.

Conditions 1) and 2) are satisfied since $\operatorname{Hom}_{\mathcal{P} \mathcal{A}(\mathcal{E})}$ is naturally a quotient object and a subobject of $\mathrm{Hom}_{\mathcal{E}}$. 
Corollary 2.6 Let the fuctor $F: \mathcal{C} \rightarrow \mathcal{D}$ satisfies the conditions $(\star)$. Then the functor $\mathcal{P} \mathcal{A}(\mathcal{F})$ defines a 1-to-1 correspondence Iso $(\mathcal{P} \mathcal{A}(\mathcal{C})) \rightarrow I$ so $(\mathcal{P} \mathcal{A}(\mathcal{D}))$ between the set of isomorphism classes of objects of the categories $\mathcal{P} \mathcal{A}(\mathcal{C})$ and $\mathcal{P} \mathcal{A}(\mathcal{D})$.

Proof: It follows immediately from Propositions 2.5 and 2.2.

Let $A^{*}(X)=A_{\operatorname{dim}(X)-*}(X)$ be the generalized oriented (co)homology theory, and $f_{0} g_{A}: A^{*} \rightarrow \mathrm{CH}^{*}$ be the morphism of oriented theories. We say that the pair $\left(A, f \circ g_{A}\right)$ satisfies the conditions $(\star, \star)$, if

(0) $A_{*}(\operatorname{Spec}(k))=A_{*}$ is zero in negative dimensions;

(1) the following map is an isomorphism

$$
f \circ g_{A} \otimes_{A_{*}} \mathbb{Z}: A_{*}(X) \otimes_{A_{*}} \mathbb{Z}=A_{*}(X) / A_{>0} \cdot A_{*}(X) \stackrel{\cong}{\rightrightarrows} \mathrm{CH}_{*}(X) .
$$

When $A^{*}(X)=\Omega^{*}(X)$ the algebraic cobordism defined by Morel and Levine, the condition $(\star, \star)$ is satisfied by [2,Theorem 14.1]. For $A^{*}(X)=$ $M G L^{2 *, *}(X)$ the condition is satisfied by Corollary 5.3. Note that $A_{*}(X)=$ $M G L^{2 d-2 *, d-*}(X)$ for $d=\operatorname{dim}(X)$, but we do not assume $A_{*}(X) \cong M G L_{2 *, *}(X)$ in this paper.

The morphism of oriented cohomology theories induces the functor between respective motivic categories, which we still denote by the same name $\operatorname{fog}_{A}: \mathrm{Cor}_{A}^{0} \rightarrow \mathrm{Cor}_{C H}^{0}$.

Proposition 2.7 If the pair $\left(A^{*}(X)\right.$, fog $\left._{A}\right)$ satisfies $(\star, \star)$, then the functor $\mathrm{fog}_{A}: \mathrm{Cor}_{A}^{0} \rightarrow \mathrm{Cor}_{C H}^{0}$ satisfies the conditions $(\star)$.

Proof: Sets of objects of both categories are identified with the set of smooth projective varieties over $k$. Thus, the condition $(\star)(0)$ is satisfied.

By definition, we can identify: $\operatorname{Hom}_{\operatorname{Cor}_{A}^{0}}([X],[Y])=A_{\operatorname{dim}(X)}(X \times Y)$, $\operatorname{Hom}_{C o r_{C H}^{0}}([X],[Y])=C H_{\operatorname{dim}(X)}(X \times Y)$, and $f_{0} g_{A}$ corresponds to the natural projection $A_{\operatorname{dim}(X)}(X \times Y) \rightarrow C H_{\operatorname{dim}(X)}(X \times Y)$. By $(\star, \star)(1)$, such projection is surjective, and the condition $(\star)(1)$ is satisfied.

The kernel $\operatorname{ker}\left(\operatorname{End}_{\mathrm{Cor}_{\mathrm{A}}^{0}}([X]) \rightarrow \operatorname{End}_{\mathrm{Cor}_{C H}^{0}}([X])\right)$ is identified with the kernel $\operatorname{ker}\left(A_{\operatorname{dim}(X)}(X \times X) \rightarrow C H_{\operatorname{dim}(X)}(X \times X)\right)$, and the latter ideal coincides with the dimension $=\operatorname{dim}(X)$ part of $A_{\geqslant 1} \cdot A_{*}(X \times X)$. Notice, that 
the composition product on $A_{*}(X \times X)$ is $A_{*}$-linear. Let $N>\operatorname{dim}(X)$. Then $k e r^{\circ N}$ is contained in the dimension $=\operatorname{dim}(X)$ part of $A_{>\operatorname{dim}(X)} \cdot A_{*}(X \times X)$. But this part is zero, since $A_{d}(Z)=0$, for any $d<0$ from condition $(\star, \star)(0)$. Thus, the condition $(\star)(2)$ is satisfied as well.

Proposition 2.7 and Corollary 2.6 imply:

Corollary 2.8 Let the pair $\left(A^{*}(X)\right.$, fog $\left._{A}\right)$ satisfies $(\star, \star)$. Then the functor $\mathrm{fog}_{A}$ defines the natural 1-to-1 correspondence Iso $\left(\mathcal{M}_{A}(k)\right) \rightarrow \operatorname{Iso}\left(\mathcal{M}_{C H}(k)\right)$ between the set of isomorphism classes of objects of $\mathcal{M}_{A}(k)$ and $\mathcal{M}_{C H}(k)$. In particular, this is so for $A^{*}=\Omega^{*}$ and $M G L^{2 *, *}$ with natural forgetful maps.

Proof: We only need to remind that $\mathcal{M}_{A}$ and $\mathcal{M}_{C H}$ are just $\mathcal{P} \mathcal{A}\left(\operatorname{Cor}_{A}^{0}\right)$ and $\mathcal{P} \mathcal{A}\left(\operatorname{Cor}_{C H}^{0}\right)$, respectively.

Let us call the corresponding motive in $\mathcal{M}_{A}$ by the same name we used to call it's projection to $\mathcal{M}_{C H}$. Thus, in $\mathcal{M}_{A}$ we have Tate-motives, Rostmotives, etc. ... . Let us denote the lifting of the Tate-motive $\mathbf{Z}(n)[2 n]$ as $\mathbf{A}(n)[2 n]$. If $U$ is arbitrary object of $\mathcal{M}_{A}(k)$, we denote $U \otimes \mathbf{A}(n)[2 n]$ as $U(n)[2 n]$. There is canonical up to sign identification: $A^{m}(U(n)[2 n])=$ $A^{m-n}(U)$.

Corollary 2.9 Let $\left(A^{*}(X), f_{\circ} g_{A}\right)$ be the pair satisfying $(\star, \star)$. Let $X$ be smooth projective cellular variety. That is, there is a filtration $X=X_{0} \supset$ $X_{1} \supset \ldots \supset X_{n}$ by closed subschemes such that for each $i, X_{i} \backslash X_{i+1}$ is a disjoint union $\coprod_{j} \mathbb{A}^{r_{j}}$ of some affine spaces. Then $M^{A}(X)$ is a direct sum of (A-) Tate-motives.

Proof: It is well-known that $M^{C H}(X)$ is a direct sum of (Chow) Tatemotives. Indeed, by the result of V.Voevodsky, $\mathcal{M}_{C H}(k)$ is a full additive subcategory of the triangulated category $D M_{g m}(k)$ (see [18]). There is a "motive with compact support" functor $M_{c}: \operatorname{Schm}(k) \rightarrow D M_{g m}(k)$ from the category of schemes of finite type over $k$ (with proper morphisms) to the category of geometric motives such that for smooth projective variety $Y$, $M_{c}(Y)=M^{C H}(Y)$ and $M_{c}\left(\mathbb{A}^{m}\right)=\mathbf{Z}(m)[2 m]$. According to [18], we have exact triangles in $D M_{g m}(k)$ :

$$
M_{c}\left(X_{i+1}\right) \rightarrow M_{c}\left(X_{i}\right) \rightarrow M_{c}\left(X_{i} \backslash X_{i+1}\right) \rightarrow M_{c}\left(X_{i+1}\right)[1] .
$$


Now using the fact that there is no hom's from $\mathbf{Z}(s)[2 s]$ to $\mathbf{Z}(t)[2 t+1]$ one easily proves by induction on $i$ that $M_{c}\left(X_{i}\right)$ is a direct sum of Tate-motives. Then, so is $M^{C H}(X)=M_{c}\left(X_{0}\right)$. Corollary 2.8 implies now that the same is true for $M^{A}(X)$.

\section{Pfister quadric and algebraic cobordisms}

Let $\alpha \in K_{n}^{M}(k) / 2$ be a pure symbol, and $Q_{\alpha}$ be the corresponding Pfister quadric of dimension $2^{n}-2$.

In the next two sections we will compute the ring of algebraic cobordisms $\Omega^{*}\left(Q_{a}\right)$ of $Q_{\alpha}$. The main (and, basically, the only) tool we are using, is the fact that the motive of this variety can be decomposed into rather simple parts. Actually, one can notice, that we could as easily start just from a quadric of height one. In particular, no quadratic form theory is used here.

We will freely use the identification $\Omega_{*}(X)=\Omega^{\operatorname{dim}(X)-*}(X)$.

By the result of M.Rost (see [11, Proposition 4]),

$$
M^{C H}\left(Q_{\alpha}\right) \cong M_{\alpha} \otimes M^{C H}\left(\mathbb{P}^{2^{n-1}-1}\right),
$$

where $M_{\alpha}$ is such motive that $\left.M_{\alpha}\right|_{\bar{k}}=\mathbf{Z} \oplus \mathbf{Z}\left(2^{n-1}-1\right)\left[2^{n}-2\right] . M_{\alpha}$ is called the Rost-motive.

By Corollary 2.8, $M^{\Omega}\left(Q_{\alpha}\right) \cong M_{\alpha}^{\Omega} \otimes M^{\Omega}\left(\mathbb{P}^{2^{n-1}-1}\right)$. We still call $M_{\alpha}^{\Omega}$ the Rost-motive, although, now it is an object of $\mathcal{M}_{\Omega}(k)$. Let $\mathbb{L}=\Omega_{*}(\operatorname{Spec}(k))$ be the Lazard ring. Denote as $\mathbf{L}(n)[2 n]$ the lift to $\mathcal{M}_{\Omega}(k)$ of the Tate motive $\mathbf{Z}(n)[2 n]$. Clearly,

$$
\left.M_{\alpha}^{\Omega}\right|_{\bar{k}}=\mathbf{L} \oplus \mathbf{L}\left(2^{n-1}-1\right)\left[2^{n}-2\right] .
$$

Then $\Omega^{*}\left(\left.M_{\alpha}^{\Omega}\right|_{\bar{k}}\right)$ can be identified with $e^{2^{n-1}-1} \cdot \mathbb{L} \oplus e_{0} \cdot \mathbb{L}$, where $e_{0} \in \Omega_{0}\left(\left.Q_{\alpha}\right|_{\bar{k}}\right)$ and $e^{2^{n-1}-1} \in \Omega^{2^{n-1}-1}\left(\left.Q_{\alpha}\right|_{\bar{k}}\right)$ satisfy: $f \circ g\left(e_{0}\right)$ is the class of a rational point, and $\operatorname{fog}\left(e^{2^{n-1}-1}\right)=h^{2^{n-1}-1}$ is the class of a plane section of codimension $2^{n-1}-1$ (thus, $\left.e^{2^{n-1}-1} \in \Omega_{2^{n-1}-1}\left(\left.M_{\alpha}^{\Omega}\right|_{\bar{k}}\right), e_{0} \in \Omega_{0}\left(\left.M_{\alpha}^{\Omega}\right|_{\bar{k}}\right)\right)$. Analogously, $\Omega^{*}\left(\left.M_{\alpha}^{\Omega}(i)[2 i]\right|_{\bar{k}}\right)$, for $0 \leqslant i \leqslant 2^{n-1}-1$ can be identified with $e^{2^{n-1}-1-i} \cdot \mathbb{L} \oplus$ $e_{i} \cdot \mathbb{L}$, and $\left\{e^{i}, e_{i}\right\}_{i=0, \ldots, 2^{n-1}-1}$ form an $\mathbb{L}$-basis of $\Omega_{*}\left(\left.Q_{\alpha}\right|_{\bar{k}}\right)$ with $f o g\left(e_{i}\right)=l_{i}$, fog $\left(e^{i}\right)=h^{i}$. Let $1 \in \Omega^{0}\left(\left.Q_{\alpha}\right|_{\bar{k}}\right)$ be the generic cycle, and $\rho$ be the projector defining $M_{\alpha}^{\Omega}\left(2^{n-1}-1\right)\left[2^{n}-2\right]$ inside $M^{\Omega}\left(Q_{\alpha}\right)$. Since $\rho(1)=e^{0}+\lambda \cdot e_{2^{n-1}-1}$, 
for certain $\lambda \in \mathbb{L}$, we can choose $e^{0}$ equal to $\rho(1)$, and it will be defined over the base field. Then the same applies to all $e^{i}, 0 \leqslant i \leqslant 2^{n-1}-1$.

Consider the map $a c^{\Omega}: \Omega^{*}(U) \rightarrow \Omega^{*}\left(\left.U\right|_{\bar{k}}\right)$. Let us denote it's image as $\bar{\Omega}^{*}(U)$.

Statement $3.1 \bar{\Omega}^{*}\left(Q_{\alpha}\right)=\left(\oplus_{i=0}^{2^{n-1}-1} \mathbb{L} e^{i}\right) \oplus\left(\oplus_{i=0}^{2^{n-1}-1} J e_{i}\right)$, where $J \subset \mathbb{L}$ is some ideal.

Proof: Really, since $e^{i}$ are defined over $k, \bar{\Omega}^{*}\left(M_{\alpha}^{\Omega}\right)=\mathbb{L} e^{2^{n-1}-1} \oplus J e_{0}$, for some ideal $J \subset \mathbb{L}$. Then $\bar{\Omega}^{*}\left(M_{\alpha}^{\Omega}(i)[2 i]\right)=\mathbb{L} e^{2^{n-1}-1-i} \oplus J e_{i}$ and the statement follows.

We need now to compute $J$.

Let us remind some facts about the Lazard ring $\mathbb{L}([13],[7])$. Let $X$ be smooth projective variety over $k$. Its characteristic numbers of degree $d$ are parametrized by partitions of $d$. That is, by sequences $\psi=\left(\psi_{1}, \ldots, \psi_{r}\right)$, where $\psi_{1} \geqslant \psi_{2} \geqslant \ldots \geqslant \psi_{r}$ and $\psi_{1}+\ldots+\psi_{r}=|\psi|=d$. To each $\psi$ one assigns smallest symmmetric polynomial $\sum_{\left(i_{1}, \ldots, i_{r}\right)} x_{i_{1}}^{\psi_{1}} \ldots x_{i_{r}}^{\psi_{r}}=R_{\psi}\left(\sigma_{1}, \ldots, \sigma_{d}\right)$ containing the monomial $x_{1}^{\psi_{1}} \ldots x_{r}^{\psi_{r}}$. Set

$$
c_{\psi}\left(-T_{X}\right)=R_{\psi}\left(c_{1}, \ldots, c_{d}\right) \in C H^{d}(X)
$$

where $c_{i}$ is the $i$-th Chern class of the (virtual) normal bundle $-T_{X}$ on $X$.

The partitions (of all degrees) parametrize a $\mathbf{Z}$-basis of the polynomial ring $\mathbf{Z}\left[b_{1}, b_{2}, \ldots\right]=\mathbf{Z}[\mathbf{b}]$ by the rule $b_{\psi}:=b_{\psi_{1}} \ldots b_{\psi_{r}}$. If we assign degree $s$ to $b_{s}$, then the degree $b_{\psi}$ will be $|\psi|$.

There is an embedding of rings $\mathbb{L} \stackrel{j}{\hookrightarrow} \mathbf{Z}[\mathbf{b}]$ defined by

$$
X \mapsto \sum_{|\psi|=\operatorname{dim}(X)} \operatorname{deg}\left(c_{\psi}\left(-T_{X}\right) \cap[X]\right) b_{\psi}
$$

where $[X] \in \mathrm{CH}^{0}(X)$ is the fundamental class of our manifold.

Denote as $I(p)$ the preimage under this embedding of the prime ideal $p \mathbf{Z}[\mathbf{b}]$. In other words, it is an ideal consisting of classes of varieties, all characteristic numbers of which are divisible by $p$. It is clearly a prime ideal. Denote as $I(p, n)$ the subideal of $I(p)$ generated by the elements of dimension $\leqslant p^{n-1}-1$. On $\mathbb{L}$ we have the action of Landweber-Novikov 
operations. By the theorem of Landweber [3], the only prime ideals stable under such operations are $I(p, n)$, for all prime $p$ and all $0 \leqslant n \leqslant \infty$ (here $I(p, \infty)=I(p))$.

There is an isomorphism $\mathbb{L} \cong \mathbf{Z}\left[x_{1}, x_{2}, \ldots\right]$, where $x_{i}$ can be chosen in such a way, that

$$
(*) \quad j\left(x_{i}\right)=\left\{\begin{array}{l}
p\left(b_{i}+\text { decomposable terms }\right), \text { if } i=p^{t}-1 \\
b_{i}+\text { decomposable terms }, \text { otherwise }
\end{array}\right.
$$

(where $p$ are prime numbers). Thus, the ideal $I(p, n)$ is generated by $x_{p^{i}-1}$, $0 \leqslant i \leqslant n$ :

$$
I(p, n)=\left(p, x_{p-1}, \ldots, x_{p^{n}-1}\right)
$$

( see Landweber [3]). In Section 5.1 bellow we will use notation $v_{i}$ for $x_{p^{i}-1}$ (when prime $p$ is fixed).

Let $X$ be smooth projective variety over $k$. Following V.Voevodsky, M.Rost, A.Merkurjev, M.Levine and F.Morel, we define ideal $I(X) \subset \mathbb{L}$ as an image of $\pi_{*}: \Omega_{*}(X) \rightarrow \Omega_{*}(\operatorname{Spec}(k))=\mathbb{L}$.

If $X$ has rational point, then, clearly, $I(X)=\mathbb{L}$.

From $(*)$ one can also obtain:

Statement 3.2 The ideal $I(2, r) \subset \mathbb{L}$ is generated by $\left[Q_{2^{i}-1}\right], 0 \leqslant i \leqslant r$, where $Q_{s}$ is quadric of dimension s.

Now we can compute $I(Q)$ for anisotropic quadric $Q$.

Theorem 3.3 Let $Q$ be anisotropic quadric such that $2^{m}-1 \leqslant \operatorname{dim}(Q)<$ $2^{m+1}-1$. Then $I(Q)=I(2, m)$.

Proof: Since $Q$ is anisotropic, $I(Q) \subset I(2)$. Really, if there is a map $X \rightarrow Q$, and some characteristic number of $X$ is odd, then on $X$ there is a zero cycle of odd degree. Then such cycle exists on $Q$, which contradicts anisotropity.

On the other hand, $\left[Q_{s}\right] \in I(Q)$, for all $0 \leqslant s \leqslant \operatorname{dim}(Q)$ (plane sections), and thus, $I(2, m) \subset I(Q)$. But, by the result of M.Levine and F.Morel, $\Omega^{*}(Q)$ as $\mathbb{L}$-module is generated by the elements of $\operatorname{dimension} \leqslant \operatorname{dim}(Q)$ and, at the same time, by the Statement 3.2, $I(2)_{<2^{m+1}-1}=I(2, m)_{<2^{m+1}-1}$. Inevitably, $I(Q)=I(2, m)$. 
Let us study the ideal $J$. Together with the quadric $Q_{\alpha}$ we can consider quadric $P_{\alpha}$ given by the smallest neighbour $p_{\alpha}$ of the Pfister form $q_{\alpha}$. By the result of M.Rost ([11, Proposition 4]), $M_{\alpha}$ is a direct summand of $M^{C H}\left(P_{\alpha}\right)$. By Corollary 2.8, $M_{\alpha}^{\Omega}$ is a direct summand of $M^{\Omega}\left(P_{\alpha}\right)$.

By the Statement 3.1, the ideal $I(2, n-1)=I\left(Q_{\alpha}\right)$ is generated by $J$ and the images of $e^{i}, 0 \leqslant i \leqslant 2^{n-1}-1$. Since the dimensions of $e^{i}$ are greater or equal $2^{n-1}-1, J \supset I(2, n-2)$.

On the other hand, by the result of M.Levine and F.Morel, $\Omega^{*}(X) / \mathbb{L}_{>0} \cdot \Omega^{*}(X) \cong C H^{*}(X)$, and thus, there is a surjection

$$
C H^{*}(X) \rightarrow \bar{\Omega}^{*}(X) / \mathbb{L}_{>0} \cdot \bar{\Omega}^{*}(X) .
$$

In particular, for $j \geqslant 2^{n-1}-1$, we get a surjection

$$
C H_{j}\left(M_{\alpha}\right) \rightarrow\left(\mathbb{L} / \mathbb{L}_{>0} \cdot \mathbb{L}\right)_{j-2^{n-1}+1} \cdot e^{2^{n-1}-1} \oplus\left(J / \mathbb{L}_{>0} \cdot J\right)_{j} \cdot e_{0} .
$$

Since $C H_{j}\left(M_{\alpha}\right)$ is a direct summand of $C H^{2^{n-1}-1-j}\left(P_{\alpha}\right)$, and the latter group is either cyclic, or trivial, we get: $\left(J / \mathbb{L}_{>0} \cdot J\right)_{j}=0$, for any $j \geqslant 2^{n-1}-1$. Hence, $J \subset I(2, n-2)$, which implies $J=I(2, n-2)$.

Theorem 3.4 Let $\alpha \in K_{n}^{M}(k) / 2$ be nonzero pure symbol, and $Q_{\alpha}$ be the corresponding Pfister quadric. Then $\bar{\Omega}^{*}\left(Q_{\alpha}\right)$ coincides with

$$
\left(\oplus_{0 \leqslant i \leqslant 2^{n-1}-1} \mathbb{L} \cdot e^{i}\right) \oplus\left(\oplus_{0 \leqslant j \leqslant 2^{n-1}-1} I(2, n-2) \cdot e_{j}\right),
$$

where $e^{i}$ is the class of the plane section of codimension $i$, and $e_{j}$ is the class of the projective subspace of dimension $j$ in $\left.Q_{\alpha}\right|_{\bar{k}}$.

Proof: We already know that $\bar{\Omega}^{*}$ has this kind of decomposition, but about $e^{i}$ and $e_{j}$ we know only that $f \circ g\left(e_{j}\right)=l_{j}, f \circ g\left(e^{i}\right)=h^{i}$, and classes $e^{i}$ are defined over $k$. Let us denote classes of plane sections and projective subspaces in $\Omega^{*}\left(\left.Q_{\alpha}\right|_{\bar{k}}\right)$ by the same symbols $h^{j}$ and $l_{i}$.

Since $e_{i}$ differs from $l_{i}$ by some $\mathbb{L}$-linear combination of $l_{0}, \ldots, l_{i-1}$, we have: $I(2, n-2)\left(\left(\oplus_{0 \leqslant m<i} l_{m}\right) \oplus e_{i}\right)=I(2, n-2)\left(\oplus_{0 \leqslant m \leqslant i} l_{m}\right)$, and one can assume that $e_{i}=l_{i}$.

The difference $e^{i}-h^{i}$ is equal to $\sum_{i<j \leqslant 2^{n-1}-1} \lambda_{j} h^{j}+\sum_{0 \leqslant m \leqslant 2^{n-1}-1} \mu_{m} l_{m}$ and is defined over the base field $k$. Then $\mu_{m} \in I(2, n-2)$ and we can substitute $e^{i}$ by $h^{i}$. 


\section{Injectivity}

The aim of this section is to show that the map $a c: \Omega^{*}\left(Q_{\alpha}\right) \rightarrow \Omega^{*}\left(\left.Q_{\alpha}\right|_{\bar{k}}\right)$ is injective, which together with the Theorem 3.4 will give a computation of $\Omega^{*}\left(Q_{\alpha}\right)$.

Remind, that we have natural maps $\mathbf{L} \stackrel{\phi}{\leftarrow} M_{\alpha}^{\Omega} \stackrel{\psi}{\leftarrow} \mathbf{L}\left(2^{n-1}-1\right)\left[2^{n}-2\right]$, which, restricted to $\bar{k}$, give the splitting $\left.M_{\alpha}^{\Omega}\right|_{\bar{k}}=\mathbf{L} \oplus \mathbf{L}\left(2^{n-1}-1\right)\left[2^{n}-2\right]$.

Lemma 4.1 Let $P$ be arbitrary subquadric of $P_{\alpha}$. Then the maps $M^{\Omega}(P) \stackrel{i d \otimes \phi}{\longleftarrow} M^{\Omega}(P) \otimes M_{\alpha}^{\Omega}$ and $\left.M^{\Omega}(P) \otimes M_{\alpha}^{\Omega} \stackrel{i d \otimes \psi}{\longleftarrow} M^{\Omega}(P)\left(2^{n-1}-1\right)\right)\left[2^{n}-2\right]$ have splittings, and

$$
M^{\Omega}(P) \otimes M_{\alpha}^{\Omega} \cong M^{\Omega}(P) \oplus M^{\Omega}(P)\left(2^{n-1}-1\right)\left[2^{n}-2\right] .
$$

Proof: Denote $d_{\alpha}:=\operatorname{dim}\left(P_{\alpha}\right)=2^{n-1}-1$, and $d:=\operatorname{dim}(P)$. Let $p_{M_{\alpha}}: M^{\Omega}\left(P_{\alpha}\right) \rightarrow M_{\alpha}^{\Omega}$ and $i_{M_{\alpha}}: M_{\alpha}^{\Omega} \rightarrow M^{\Omega}\left(P_{\alpha}\right)$ be the maps defining the Rost motive as the direct summand of the motive of $P_{\alpha}$. The splitting $s_{P}: M^{\Omega}(P) \rightarrow M^{\Omega}(P) \otimes M_{\alpha}^{\Omega}$ is given by the composition:

$$
M^{\Omega}(P) \stackrel{\Delta}{\longrightarrow} M^{\Omega}(P \times P) \stackrel{i d \otimes e m b}{\longrightarrow} M^{\Omega}\left(P \times P_{\alpha}\right) \stackrel{i d \otimes p_{M_{\alpha}}}{\longrightarrow} M^{\Omega}(P) \otimes M_{\alpha}^{\Omega} .
$$

It follows just from the fact that the composition $M^{\Omega}\left(P_{\alpha}\right) \stackrel{p_{M_{\alpha}}}{\rightarrow} M_{\alpha}^{\Omega} \stackrel{\phi}{\rightarrow} \mathbf{L}$ is induced by the projection $P_{\alpha} \rightarrow \operatorname{Spec}(k)$. Analogously, the splitting $t_{P}$ : $M^{\Omega}(P) \otimes M_{\alpha}^{\Omega} \rightarrow M^{\Omega}(P)\left(d_{\alpha}\right)\left[2 d_{\alpha}\right]$ is given by the composition:

$$
\begin{array}{r}
M^{\Omega}(P) \otimes M_{\alpha}^{\Omega} \stackrel{i d \otimes i_{M_{\alpha}}}{\longrightarrow} M^{\Omega}\left(P \times P_{\alpha}\right) \stackrel{(i d \otimes e m b)^{\vee}}{\longrightarrow} M^{\Omega}(P \times P)\left(d_{\alpha}-d\right)\left[2\left(d_{\alpha}-d\right)\right] \\
\stackrel{\Delta^{\vee}}{\longrightarrow} M^{\Omega}(P)\left(d_{\alpha}\right)\left[2 d_{\alpha}\right],
\end{array}
$$

where for $\varphi: M(X) \rightarrow M(Y)(r)[2 r]$ represented by the map $W \rightarrow X \times Y$, the dual map $\varphi^{\vee}: M(Y) \rightarrow M(X)(d+r)[2(d+r)]$ is represented by the composition $W \rightarrow X \times Y \stackrel{s_{1,2}}{\rightarrow} Y \times X$ (here $\left.d=\operatorname{dim}(Y)-\operatorname{dim}(X)\right)$.

Since $(i d \otimes \phi) \circ(i d \otimes \psi)=0, M^{\Omega}(P) \otimes M_{\alpha}^{\Omega}$ contains direct summand $M^{\Omega}(P) \oplus M^{\Omega}(P)\left(d_{\alpha}\right)\left[2 d_{\alpha}\right]$. Let $N$ be the complimentary summand. In view of Corollary 2.8, to prove that $N=0$, it is sufficient to show that $f \circ g(N) \in$ $\mathcal{M}_{C H}(k)$ is zero.

Since $\left.M_{\alpha}^{C H}\right|_{\bar{k}}=\mathbf{Z} \oplus \mathbf{Z}\left(2^{n-1}-1\right)\left[2^{n}-2\right]$, the restriction $\left.f \circ g(N)\right|_{\bar{k}}$ is zero. But it follows from the Rost Nilpotence Theorem (see [12, Proposition 9]) 
that the motive of the product of quadrics does not contain phantom direct summands, that is, nonzero direct summands which become zero when restricted to $\bar{k}$. Thus, $N=0$.

Remark: In [12, Proposition 9] the Nilpotence Theorem is formulated only for the case of motives of quadrics, but (some modification of) the proof works as well for the motives of products of quadrics (cf. the proof of [1, Theorem 8.2]). Another proof of Lemma 4.1 can be easily obtained from the fact that, in the category $D M^{e f f}(k), M_{\alpha}^{C H}$ can be presented in the form Cone $[-1]\left(M\left(\check{C}\left(Q_{\alpha}\right)\right) \rightarrow M\left(\check{C}\left(Q_{\alpha}\right)\right)\left(2^{n-1}-1\right)\left[2^{n}-1\right]\right)$ (see [19]).

Lemma 4.2 Let $R$ be smooth projective variety of dimension $d<2^{n-1}-$ 1 such that the projection $M^{\Omega}(R) \leftarrow M^{\Omega}(R) \otimes M_{\alpha}^{\Omega}$ has a splitting $s$ and $M^{\Omega}(R) \otimes M_{\alpha}^{\Omega} \cong M^{\Omega}(R) \oplus M^{\Omega}(R)\left(2^{n-1}-1\right)\left[2^{n}-2\right]$. Then such splitting is unique.

Proof: The uniqueness follows from the fact that

$$
\operatorname{Hom}_{\mathcal{M}_{\Omega}}\left(M^{\Omega}(R), M^{\Omega}(R)\left(2^{n-1}-1\right)\left[2^{n}-2\right]\right)=\Omega^{d+2^{n-1}-1}(R \times R)=0,
$$

by the dimensional considerations.

Let $Q_{2^{i}-1}$ be a subquadric of $P_{\alpha}$ of dimension $2^{i}-1$, where $0 \leqslant i \leqslant n-2$. Let $\pi_{i}: M^{\Omega}\left(Q_{2^{i}-1}\right) \otimes M_{\alpha}^{\Omega} \rightarrow M_{\alpha}^{\Omega}$ be the projection, and $s_{i}: M^{\Omega}\left(Q_{2^{i}-1}\right) \rightarrow$ $M^{\Omega}\left(Q_{2^{i}-1}\right) \otimes M_{\alpha}^{\Omega}$ be the splitting from Lemma 4.1. Applying Lemma 4.2 to the case $R=Q_{2^{i}-1}$, we get that $s_{i}$ is unique.

Denote as $c_{i}$ the element $\left(\pi_{i}\right)_{*}\left(s_{i}\right)_{*}\left(\left[1_{Q_{2^{i}-1}}\right]\right) \in \Omega_{2^{i}-1}\left(M_{\alpha}^{\Omega}\right)$. Notice, that $c_{i}$ is just $p_{M_{\alpha *}}\left(\left[Q_{2^{i}-1} \subset P_{\alpha}\right]\right)$, where $\left[Q_{2^{i}-1} \subset P_{\alpha}\right] \in \Omega_{2^{i}-1}\left(P_{\alpha}\right)$ is the class of our subquadric. These classes satisfy the following equations:

Proposition $4.3 c_{i} \cdot\left[Q_{2^{j}-1}\right]=c_{j} \cdot\left[Q_{2^{i}-1}\right]$, where $\left[Q_{r}\right] \in \Omega_{r}(\operatorname{Spec}(k))=\mathbb{L}$ is the class of the quadric of dimension $r$.

Proof:

Consider the diagram:

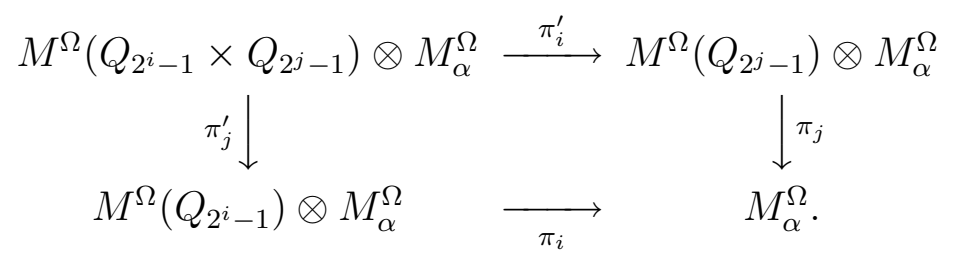


From Lemma 4.1 it follows that the variety $R=Q_{2^{i}-1} \times Q_{2^{j}-1}$ satisfies the conditions of Lemma 4.2. (Indeed, if variety $X$ satisfies these conditions, then variety $X \times Y$ satisfies them too (for any $Y$ ) - just multiply the old splitting by $i d_{Y}$.) It follows from Lemma 4.2 that the splittings $s_{i} \otimes i d_{M^{\Omega}\left(Q_{2^{j}-1}\right)}$ and $s_{j} \otimes i d_{M^{\Omega}\left(Q_{2^{i}-1}\right)}$ coincide and are equal to the unique splitting $s$ for the variety $Q_{2^{i}-1} \times Q_{2^{j}-1}$. Consequently, $\left(\pi_{j}^{\prime}\right)^{*}\left(s_{i}\right)_{*}(1)=s_{*}(1)=\left(\pi_{i}^{\prime}\right)^{*}\left(s_{j}\right)_{*}(1)$, and

$$
\begin{array}{r}
c_{i} \cdot\left[Q_{2^{j}-1}\right]=\left(\pi_{j}\right)_{*}\left(\pi_{j}\right)^{*}\left(c_{i}\right)=\left(\pi_{j}\right)_{*}\left(\pi_{j}\right)^{*}\left(\pi_{i}\right)_{*}\left(s_{i}\right)_{*}(1)= \\
\left(\pi_{j}\right)_{*}\left(\pi_{i}^{\prime}\right)_{*}\left(\pi_{j}^{\prime}\right)^{*}\left(s_{i}\right)_{*}(1)=\left(\pi_{j}\right)_{*}\left(\pi_{i}^{\prime}\right)_{*}\left(\pi_{i}^{\prime}\right)^{*}\left(s_{j}\right)_{*}(1)= \\
\left(\pi_{j}\right)_{*}\left(s_{j}\right)_{*}(1) \cdot\left[Q_{2^{i}-1}\right]=c_{j} \cdot\left[Q_{2^{i}-1}\right] .
\end{array}
$$

Let $A \subset \Omega^{*}\left(M_{\alpha}^{\Omega}\right)$ be the ideal generated by the elements $c_{i}, 0 \leqslant i \leqslant$ $n-2$ and by $e^{2^{n-1}-1}$ (the generic cycle of the Rost motive). Consider $\bar{A} \subset$ $\Omega^{*}\left(\left.M_{\alpha}^{\Omega}\right|_{\bar{k}}\right)=\mathbb{L} \cdot e_{0} \oplus \mathbb{L} \cdot e^{2^{n-1}-1}$ - the image of $A$ under the natural extension of scalars map ac. Since $c_{i}$ is just $p_{M_{\alpha *}}\left(\left[Q_{2^{i}-1} \subset P_{\alpha}\right]\right)$, clearly, $a c\left(c_{i}\right)=$ $\left[Q_{2^{i}-1}\right] \cdot e_{0}$. Because $\mathbb{L}$ is a unique factorization domain, it follows from Proposition 4.3 that the map $a c: A \rightarrow \bar{A}$ is an isomorphism.

The Chow groups of $M_{\alpha}^{C H}$ were computed by M.Rost in [11]. His result is:

$$
C H^{*}\left(M_{\alpha}^{C H}\right)=\mathbb{Z} \cdot f o g\left(c_{0}\right) \oplus \mathbb{Z} \cdot e^{2^{n-1}-1} \oplus\left(\oplus_{i=1}^{n-2} \mathbb{Z} / 2 \cdot \operatorname{fog}\left(c_{i}\right)\right) .
$$

This shows that the composition $A \rightarrow \Omega^{*}\left(M_{\alpha}^{\Omega}\right) \rightarrow \mathrm{CH}^{*}\left(M_{\alpha}^{C H}\right)$ is surjective. Since $C H^{*}\left(M_{\alpha}^{C H}\right)=\Omega^{*}\left(M_{\alpha}^{\Omega}\right) / \mathbb{L}^{<0} \Omega^{*}\left(M_{\alpha}^{\Omega}\right)$, and $\Omega^{*}\left(M_{\alpha}^{\Omega}\right)$ is a positively (by dimension) graded module, we get $A=\Omega^{*}\left(M_{\alpha}^{\Omega}\right)$. So, we have proved:

Proposition 4.4 The map ac: $\Omega^{*}\left(Q_{\alpha}\right) \rightarrow \Omega^{*}\left(\left.Q_{\alpha}\right|_{\bar{k}}\right)$ is injective, and Theorem 3.4 describes the $\Omega^{*}\left(Q_{\alpha}\right)$.

Remark: It should be noticed, that an alternative proof of Proposition 4.4 can be obtained with the help of symmetric operations (see [16] and [15]). It has an advantage of being independent from the computations of [11]. In turn, it gives a new way to compute the Chow groups of a Pfister quadric.

Recall the motivic cobordism theory $M G L^{*, *}(X)$ defined by V.Voevodsky. It follows from Corollary 5.2 bellow and the naturality of the realization map 
$t_{\mathbb{C}}$, that $M G L^{-2 *,-*}(p t)=.\mathbb{L}_{*}=\Omega^{-*}(p t$.$) . Moreover by the universality of$ $\Omega^{*}(X)$ for oriented cohomologies, there is the natural map ([5])

$$
\rho_{M G L}: \Omega^{*}(X) \rightarrow M G L^{2 *, *}(X)
$$

which is epimorphic from Corollary 5.2, and is monomorphic for $X=\left.Q_{\alpha}\right|_{\bar{k}}$ from Corollary 2.9. Hence we get:

Corollary 4.5 $\Omega^{*}\left(Q_{\alpha}\right) \cong M G L^{2 * * *}\left(Q_{\alpha}\right)$.

Infact, almost all arguments in Sections 3,4 work also for $M G L^{2 * * *}(X)$. However, in the next sections we will give another method of computing this ring by means of the Atiyah-Hirzebruch spectral sequence.

\section{Atiyah-Hirzebruch spectral sequence}

In this section we consider the Atiyah-Hirzebruch spectral sequences (AHss) for generalized motivic cohomologies related to the spectrum $M G L^{*, *}(X)$ constructed by Voevodsky ([17]). Since we are using the cohomology operations, below we mainly work with cohomology theories.

In the current section, $p$ is a fixed prime number. Let $k$ be a subfield of the complex number field $\mathbb{C}$, and $t_{\mathbb{C}}$ be the induced realization map (see $[17])$. Recall the complex cobordism ring ([13])

$$
\mathbb{L}_{-*} \cong M U^{2 *}(p t .)=M U^{2 *} \cong \mathbb{Z}\left[x_{1}, x_{2}, \ldots\right], \quad \operatorname{deg}\left(x_{i}\right)=\left|x_{i}\right|=-2 i .
$$

We have the map $i: \mathbb{L}_{-*} \cong M U^{2 *} \rightarrow M G L^{2 *, *}\left(p t\right.$.) such that $t_{\mathbb{C}} i$ is the identity on $M U^{2 *}$. Moreover $M U^{2 *} \cong M G L^{2 * *}(p t$.) by Corollary 5.2 bellow and the naturality of $t_{\mathbb{C}}$.

Let us denote as $A M U$ the spectrum $M G L_{(p)}$ representing the motivic cobordism theory, i.e., $M G L^{*, *}(-)_{(p)}=A M U^{*, *}(-)$. Of course, $t_{\mathbb{C}}(A M U)=$ $M U_{(p)}$. Since $M G L^{* * *}(X)$ is a multiplicative cohomology theory, we know it is an $M G L^{*, *}\left(p t\right.$.)-module, and hence, an $M U^{*}$-module. Given a regular sequence $S_{n}=\left(s_{1}, \ldots, s_{n}\right)$ with $s_{i} \in M U_{(p)}^{*}$, we can inductively construct the $A M U$-module spectrum by the cofibering of spectra

$$
\mathbb{T}^{-1 / 2\left|s_{i}\right|} \wedge A M U\left(S_{i-1}\right) \stackrel{\times s_{i}}{\longrightarrow} A M U\left(S_{i-1}\right) \rightarrow A M U\left(S_{i}\right)
$$


where $\mathbb{T}=S_{s}^{1} \wedge S_{t}^{1}$ so that $M(\mathbb{T})=\mathbb{Z}(1)[2]$. Hence we get the generalized cohomology theory $A M U\left(S_{n}\right)^{*, *}(X)$ such that

$$
t_{\mathbb{C}}\left(A M U\left(S_{n}\right)\right) \cong M U\left(S_{n}\right) \text { with } M U\left(S_{n}\right)^{*}=M U^{*} /\left(\operatorname{Ideal}\left(S_{n}\right)\right) .
$$

This way, we can construct spectra $A B P, A k(n), A H \mathbb{Z}, A H \mathbb{Z} / p$ so that $t_{\mathbb{C}}(A h) \cong h$ for $h=B P, k(n), \ldots$. Recall that $B P=M U\left(x_{i} \mid i \neq p^{j}-1\right)_{(p)}$ and $B P^{*}=\mathbb{Z}_{(p)}\left[v_{1}, v_{2}, \ldots\right]$, where we denote $x_{p^{i}-1}$ as $v_{i}$.

Remark. It follows from the Statement 3.2, that in the case $p=2$ we can take $v_{i}=\left[Q_{2^{i}-1}\right]$.

One of important results for these cohomology theories is that in the $\mathbb{A}^{1}$-stable homotopy category, we get $H_{\mathbb{Z} / p} \cong A H \mathbb{Z} / p=A M U\left(p, x_{1}, x_{2}, \ldots\right)$, e.g., $A H \mathbb{Z} / p^{*, *}(X) \cong H^{*, *}(X ; \mathbb{Z} / p)$. Direct application of this fact is the construction of AHss for the connected Morava $K$-theory $A k(n)^{*, *}(-)$ (here $\left.k(n)^{*}=\mathbb{Z} / p\left[v_{n}\right]\right)$. The above isomorphism induces the cofibering

$$
\mathbb{T}^{p^{n}-1} \wedge A k(n) \stackrel{v_{n}}{\longrightarrow} A k(n) \longrightarrow A H \mathbb{Z} / p=H_{\mathbb{Z} / p}
$$

This induces the exact couple and the spectral sequence (AHss)

$$
E_{2}^{*, *, *} \cong H^{*, *}(X ; \mathbb{Z} / p) \otimes k(n)^{*} \Longrightarrow A k(n)^{*, *}(X) \text {. }
$$

If $S \subset\left(p, x_{1}, \ldots, x_{n}, \ldots\right)$, then we have the natural map of spectra

$$
A M U(S) \rightarrow A M U(p, . .)=H \mathbb{Z} / p \quad\left(A M U(S) \rightarrow H \mathbb{Z}_{(p)} \quad \text { if } p \notin S\right) .
$$

In such a case, we can construct AHss. (The $M G L$ case of this sequence was constructed by Hopkins and Morel.)

Theorem 5.1 ([23]) Let $A h=A M U(S)$ for some regular sequence of generators $S=\left(a_{1}, \ldots\right), a_{j}=x_{i_{j}} \in M U_{(p)}^{*}$. Then there is the (natural $M U_{(p)}^{*}$ module) Atiyah-Hirzebruch spectral sequence

$$
E(A h)_{2}^{\left(m, n, 2 n^{\prime}\right)}=H^{m, n}\left(X ; h^{2 n^{\prime}}\right) \Longrightarrow A h^{m+2 n^{\prime}, n+n^{\prime}}(X)
$$

with the differential $\quad d_{2 r+1}: E_{2 r+1}^{\left(m, n, 2 n^{\prime}\right)} \rightarrow E_{2 r+1}^{\left(m+2 r+1, n+r, 2 n^{\prime}-2 r\right)}$.

\section{Remarks.}


1) The cohomology $H^{m, n}\left(X, h^{2 n^{\prime}}\right)$ here is the usual motivic cohomology with coefficients in the abelian group $h^{2 n^{\prime}}$, e.g., if $h^{2 n^{\prime}}$ is $\mathbb{Z} / p$-module, then $H^{m, n}\left(X ; h^{2 n^{\prime}}\right) \cong H^{m, n}(X ; \mathbb{Z} / p) \otimes h^{2 n^{\prime}}$. In particular, if $X$ is smooth, then $E_{r}^{m, n, 2 n^{\prime}} \cong 0$ for $m>2 n$.

2) The convergence in AHss means that there is the filtration

$$
A h^{*, *^{\prime}}(X)=F_{0}^{*, *^{\prime}} \supset F_{1}^{*, *^{\prime}} \supset F_{2}^{*, *^{\prime}} \supset \ldots
$$

such that $F_{i}^{*, *^{\prime}} / F_{i+1}^{*, *^{\prime}} \cong E_{\infty}^{*+2 i, *^{\prime}+i,-2 i}$.

3) Let $S=\left(x_{i_{1}}, \ldots\right) \subset R=\left(x_{j_{1}}, \ldots\right)$. Then the induced map $A M U(S) \rightarrow$ $A M U(R)$ of spectra induces the natural $M U_{(p)}^{*}$ - module map of AHss

$$
E(A M U(S))_{r}^{*, *, *} \rightarrow E(A M U(R))_{r}^{*, *, *}
$$

Let us denote first.deg as [deg], the second.deg as (deg), and, finally, denote

$$
w(x)=-[\operatorname{deg}](x)+2(\operatorname{deg})(x)
$$

so that $w(x) \geq 0$ for nonzero element $x$ in the spectral sequences (or cohomology theories) for a smooth $X$. Moreover, note that $w\left(d_{r}\right)=-1$ and third.deg $\left(d_{r}\right)=-r+1<0$.

Corollary 5.2 If $h^{0} \cong \mathbb{Z}_{(p)}$, then for any smooth $X$, the natural map

$$
A h^{2 * * *}(X) \otimes_{M U_{(p)}^{*}} \mathbb{Z}_{(p)} \cong C H^{*}(X)_{(p)}
$$

is an isomorphism

Proof: First note, that $E_{2}^{*, *^{\prime}, 0} \cong H^{*, *^{\prime}}(X)_{(p)} \otimes h^{0} \cong H^{*, *^{\prime}}(X)_{(p)}$. In particular, we can identify $E_{2}^{2 *, * 0} \cong C H^{*}(X)_{(p)}$. For element $x \in E^{2 *, *, *^{\prime \prime}}$ (i.e., $w(x)=$ 0 ), we have $d_{r}(x)=0$ since $w\left(d_{r}(x)\right)=-1<0$. Thus, $E_{r}^{2 *, * *^{\prime \prime}}$ is a quotient of $E_{2}^{2 *, *, *^{\prime \prime}}$. But we also know that $\operatorname{Im}\left(d_{r}\right) \subset E_{r}^{*, *^{\prime},<0}$. Hence,

$$
E_{2}^{2 *, *, 0}=E_{\infty}^{2 *, *, 0} \cong F_{0}^{2 *, *} / F_{1}^{2 *, *} \subset \operatorname{gr} A h^{2 *, *}(X)
$$

where we identify $E_{\infty}^{*, *, *}$ with $g r A h^{*, *}(X)$. The isomorphism $h^{<0} E_{\infty}^{2 *, *, 0}=$ $E_{\infty}^{2 *, *,<0}$ is $h^{<0}\left(F_{0}^{2 *, *} / F_{1}^{2 *, *}\right)=\oplus_{i \geq 1} F_{i}^{2 *, *} / F_{i+1}^{2 *, *}$ by the definition of the filtration (2). Of course $F_{i} \supset F_{i+1}$, we see $h^{<0} F_{0}^{2 *, *}=F_{1}^{2 *, *}$. Thus we have $A h^{2 *, *}(X) \otimes_{M U^{*}} \mathbb{Z}_{(p)} \cong F_{0}^{2 *, *} / F_{1}^{2 *, *} \cong C H^{*}(X)_{(p)}$. 
Corollary 5.3 Let $A h^{2 *, *}(X)$ be generalized cohomology theory from Theorem 5.1. Moreover, let $A h^{* * *}(X)$ be multiplicative and $h^{0} \cong \mathbb{Z}_{(p)}$. Then the condition $(\star, \star)$ from Section 2 is satisfied for $A^{*}(X)=A h^{2 * * *}(X)$ and the natural functor fog $_{A}: A^{*} \rightarrow \mathrm{CH}_{(p)}^{*}$.

Proof: The condition $(\star, \star)(0)$ is trivial, and the condition $(\star, \star)(1)$ follows from the fact that $A h^{2 *, *}(X) \otimes_{M U_{(p)}^{*}} \mathbb{Z}_{(p)} \rightarrow C H^{*}(X)_{(p)}$ can be decomposed into the composition $A h^{2 *, *}(X) \otimes_{M U_{(p)}^{*}} \mathbb{Z}_{(p)} \stackrel{\pi}{\rightarrow} A h^{2 *, *}(X) \otimes_{A_{(p)}^{*}} \mathbb{Z}_{(p)} \rightarrow$ $C H^{*}(X)_{(p)}$, where $\pi$ is surjective.

Hence $M G L^{2 *, *}(X)_{(p)}$ satisfy $(\star, \star)$ and so does $A B P^{2 *, *}(X)$ by the following reason (while for $p=2, A k(n)^{2 *, *}(X)$ is not a multiplicative cohomology theory). We can define $([22,23])$ the multiplicative projection $\Phi: A M U \rightarrow A M U$ such that $\Phi\left(x_{i}\right)=x_{i}$ if $i=p^{j}-1$ and $\Phi\left(x_{i}\right)=0$ otherwise. Then we can show ( (4.5) in [23]) $A B P \cong \Phi A M U$. Moreover we have

Lemma 5.4 For all $X$, we have a $B P^{*}$-algebra isomorphism

$$
M G L^{*, *}(X)_{(p)} \cong A B P^{*, *}(X) \otimes_{B P^{*}} M U_{(p)}^{*} .
$$

To see the differentials of AHss, we recall the cohomology operations in mod $p$ motivic cohomology. In this cohomology, we have the Bockstein homomorphism $\beta$ and the reduced powers operations $P^{i}$ which commute with the realization map $t_{\mathbf{C}}$. Moreover, we have the Milnor operation

$$
Q_{i}: H^{*, *}(X ; \mathbb{Z} / p) \rightarrow H^{*+2 p^{i}-1, *+p^{i}-1}(X ; \mathbb{Z} / p)
$$

with $Q_{0}=\beta$ and $Q_{i+1}=\left[Q_{i}, P^{p^{i}}\right] \bmod (\rho)$ where $\rho=-1 \in k^{*} /\left(k^{*}\right)^{p}=$ $H^{1,1}(p t . ; \mathbb{Z} / p)$ (see [20] for details). We note $Q_{i}^{2}=0$ and $Q_{i} Q_{j}=-Q_{j} Q_{i}$. But $Q_{i}$ is not a derivation when $\rho \neq 0$. We also note that $w\left(P^{i}\right)=0$ and $w\left(Q_{i}\right)=-1$.

V.Voevodsky (in particular, Lemma 2.2 in [21]) and G.Powell show that the $\bmod p$ motivic Steenrod algebra $A_{p}^{*, *}$ is generated as an $H^{*, *}(p t, \mathbb{Z} / p)$ module by the products of $P^{i}$ and $\beta$. In particular, they also prove that as a left $H^{*, *}(p t ; \mathbb{Z} / p)$-module,

$$
A_{p}^{*, *} \cong H^{*, *}(p t ; \mathbb{Z} / p) \otimes R P \otimes \Lambda\left(Q_{0}, Q_{1}, \ldots\right)
$$


where $R P$ is the $\mathbb{Z} / p$-module generated by products of reduced powers $P^{i_{1}} \ldots P^{i_{n}}$ (without the Bockstein).

Recall $k(n)^{*} \cong \mathbb{Z} / p\left[v_{n}\right]$. Since $\left|v_{n}\right|\left(=\operatorname{deg}\left(v_{n}\right)\right)=-2\left(p^{n}-1\right)$, we know that $k(n)^{*}=0$ for $* \neq 0 \bmod \left(2 p^{n}-2\right)$. So we have

$$
E_{2}^{*, *^{\prime}, *^{\prime \prime}}=H^{*, *^{\prime}}(X ; \mathbb{Z} / p) \otimes k(n)^{*^{\prime \prime}}=0 \quad \text { for } *^{\prime \prime} \neq 0 \bmod \left(2 p^{n}-2\right)
$$

Hence the first nonzero differential is $d_{2 p^{n}-1}$.

By the naturality, the first nonzero differential

$$
d_{2 p^{n}-1}: H^{*, *}(X ; \mathbb{Z} / p) \rightarrow H^{*+2 p^{n}-1, *+p^{n}-1}(X ; \mathbb{Z} / p) \otimes v_{n}
$$

must be a cohomology operation of the motivic cohomology. (Cohomology operations are just natural transformations of the cohomology theory.) We know $([22,23]) d_{2 p^{n}-1}(x)=v_{n} \otimes Q_{n}(x)$ for AHss of (the topological) $k(n)^{*}$ theory. From (3), we have

Lemma 5.5 The first nonzero differential in AHss for $A k(n)^{*, *}(X)$ is given by

$$
d_{2 p^{n}-1}(x)=v_{n} \otimes\left(Q_{n}+a_{I J} P^{I} Q_{J}\right)(x)
$$

where $P^{I} \in R P, Q_{J} \in \Lambda\left(Q_{0}, \ldots, Q_{n-1}\right),|J| \geq 2, a_{I J} \in H^{>0, *}(p t . \mathbb{Z} / p)$.

\section{$6 M G L^{2 *, *}$ theory of Pfister quadrics}

Let $\check{C}$ ech complex $\check{C}(X)$ be the simplicial scheme such that $\check{C}(X)_{n}=X^{n+1}$ and the faces and degeneracy maps are given by partial projections and diagonals respectively $([17,19])$. In the stable $\mathbb{A}^{1}$ homotopy category, define $\tilde{C}(X)$ by the cofiber sequence $\tilde{C}(X) \rightarrow \check{C}(X) \rightarrow \operatorname{Spec}(k)$.

Let us write $\check{C}\left(Q_{\alpha}\right)=\chi_{\alpha}$ and $\tilde{C}\left(Q_{\alpha}\right)=\tilde{\chi}_{a}$. V.Voevodsky proved that in the triangulated category of motives $D M_{-}^{e f f}$ (see [19] for details ), there exists the distinguished triangle

$$
M\left(\chi_{\alpha}\right)\left(2^{n-1}-1\right)\left[2^{n}-2\right] \rightarrow M_{\alpha} \rightarrow M\left(\chi_{\alpha}\right) \stackrel{\delta_{a}}{\rightarrow} M\left(\chi_{\alpha}\right)\left(2^{n-1}-1\right)\left[2^{n}-1\right] .
$$

Using the induced long exact sequence of motivic cohomologies and the arguments from the paper [9], we can prove 
Theorem 6.1 ([23]) There is a $K_{*}^{M}(k) \otimes \Lambda\left(Q_{0}, \ldots, Q_{n-1}\right)$-modules isomorphism

$$
H^{*, *}\left(\tilde{\chi}_{a} ; \mathbb{Z} / 2\right) \cong K_{*}^{M}(k) /(\operatorname{Ker}(\alpha)) \otimes \Lambda\left(Q_{0}, \ldots, Q_{n-1}\right) \otimes \mathbb{Z} / 2\left[\delta_{a}^{2}\right]\left\{\alpha^{\prime}\right\}
$$

where $\operatorname{deg}\left(\delta_{a}^{2}\right)=\left(2^{n+1}-2,2^{n}-2\right), \operatorname{deg}\left(\alpha^{\prime}\right)=(n, n-1)$.

Corollary 6.2 There is a $K_{*}^{M}(k)$-module isomorphism for $[\mathrm{deg}]-(\mathrm{deg})>0$ and $[d e g] \leq 2^{n}-2$,

$$
H^{*, *}\left(M_{\alpha} ; \mathbb{Z} / 2\right) \cong K_{*}^{M}(k) /(\operatorname{Ker}(\alpha)) \otimes \Lambda\left(Q_{0}, \ldots, Q_{n-2}\right)\left\{\alpha^{\prime}\right\} .
$$

Let $\tau$ be the nonzero element in $H^{0,1}(p t$.; $\mathbb{Z} / 2) \cong \mathbb{Z} / 2$. Then the elements $\alpha$ and $\alpha^{\prime}$ are related by: $\tau \alpha^{\prime}=\pi^{*}(\alpha)$ in $H^{n, n}\left(M_{\alpha} ; \mathbb{Z} / 2\right)([23])$, where we denote by $\pi^{*}$ the composition $K_{n}^{M}(k) / 2 \cong H^{n, n}(p t . ; \mathbb{Z} / 2) \stackrel{\pi^{*}}{\rightarrow} H^{n, n}\left(M_{\alpha} ; \mathbb{Z} / 2\right)$.

Note that $w\left(\alpha^{\prime}\right)=n-2$. Since $w\left(Q_{i}\right)=-1$, if we apply $n-2$-times various operations $Q_{i}$ to $\alpha^{\prime}$, then the weight of the result will be just zero. Indeed the $w t(x)=0$ parts of $H^{2 *, *}\left(M_{\alpha} ; \mathbb{Z} / 2\right)$ for $0<* \leq 2^{n}-2$ is expressed as $Q_{0} \ldots \hat{Q}_{i} \ldots Q_{n-2}\left(\alpha^{\prime}\right)$. Moreover $c_{i}$ is the Bockstein $\left(=Q_{0}\right)$ image for $i>0$ implies the result of Rost - see equation (2) of Section 4.

Corollary 6.3 (Rost [12]) We have an isomorphism

$$
C H^{*}\left(M_{\alpha}\right) \cong H^{2 * * *}\left(M_{\alpha} ; \mathbb{Z}\right) \cong \mathbb{Z}\left\{1, c_{0}\right\} \oplus \mathbb{Z} / 2\left\{c_{1}, \ldots, c_{n-2}\right\}
$$

where $c_{i}$ is a lift of $Q_{0} \ldots \hat{Q}_{i} \ldots Q_{n-2}\left(\alpha^{\prime}\right) \in H^{2 *, *}\left(M_{\alpha} ; \mathbb{Z} / 2\right)$ and $\operatorname{deg}\left(c_{i}\right)=\left(2^{n}-\right.$ $\left.2^{i+1}, 2^{n-1}-2^{i}\right)$.

Here we recall that the Rost motive $M_{\alpha}$ can be represented as a motive of some affine quadric. For a quadratic form $\xi$ let us denote as $Q_{\xi}$ the quadric defined by $\xi$, e.g., $Q_{\alpha}=Q_{\phi_{\alpha}}$.

Theorem 6.1 (Rost [11], Hoffmann [2]) Let $\xi$ be a subform of the Pfister form $\phi_{\alpha}$ of $\operatorname{dim}(\xi)=2^{n-1}+s, s>0$ (i.e., $\xi$ is a neighbour of $\phi_{\alpha}$ ). Let $\eta$ be the complementary form $\left(\phi_{\alpha}=\xi \oplus \eta\right)$. Then

$$
M\left(Q_{\xi}\right)=M_{\alpha} \otimes M\left(\mathbb{P}^{s-1}\right) \oplus M\left(Q_{\eta}\right) \otimes \mathbb{T}^{\otimes s} .
$$


Proof: Rost proved (Proposition 2.4 of [11]) that the result holds if there is a form $\eta$ over $k$ such that $\left.\eta\right|_{k\left(\phi_{\alpha}\right)}=\left(\left.\xi\right|_{k\left(\phi_{\alpha}\right)}\right)_{\text {anis. }}$. One could take $\eta$ to be a complementary form since $\left.\phi_{\alpha}\right|_{k\left(\phi_{\alpha}\right)}$ is hyperbolic. Here we see $\left.\eta\right|_{k\left(\phi_{\alpha}\right)}$ is anisotropic by the following result of Hoffmann (Theorems 1 in [2]). If $q, q^{\prime}$ are anisotropic forms over $k$ such that $\operatorname{dim}(q)>2^{n-1}>\operatorname{dim}\left(q^{\prime}\right)$, then $\left.q^{\prime}\right|_{k(q)}$ is anisotropic.

One of direct corollaries of this theorem is $M\left(Q_{\alpha}\right) \cong M_{\alpha} \otimes M\left(\mathbb{P}^{2^{n-1}-1}\right)$ (as stated in (1) in Section 3). Moreover let $Q_{\xi}$ be a quadric defined by subform of $\phi_{\alpha}$ of codimension 1 . Then we see $Q_{\xi} \cong M_{\alpha} \otimes M\left(\mathbb{P}^{2^{n-1}-2}\right)$.

Corollary 6.4 The Rost motive $M_{\alpha}$ is represented as the motive of affine quadric $U_{\alpha}=Q_{\phi_{a}}-Q_{\xi}$.

Proof: In $D M(k)$ there is the distinguished triangle

$$
M\left(U_{\alpha}\right) \rightarrow M\left(Q_{\alpha}\right) \stackrel{f}{\rightarrow} M\left(Q_{\xi}\right)(1)[2] \rightarrow M\left(U_{\alpha}\right)[1] .
$$

Hence the corollary follows from the fact that $M\left(Q_{\alpha}\right) \cong \oplus_{i=0}^{2^{n-1}-1} M_{\alpha}(i)[2 i]$, $M\left(Q_{\xi}\right)(1)[2] \cong \oplus_{i=1}^{2^{n-1}-1} M_{\alpha}(i)[2 i]$, and the map $f$ defines an isomorphism of the respective summands $M_{\alpha}(i)[2 i]$ (by the Rost Nilpotence Theorem, since it is an isomorphism over the algebraic closure).

Let $A^{*, *^{\prime}}(X)$ be any (oriented) generalized cohomology theory. Then for smooth $X$ and regular closed $Z \subset X$ of codimension $c$ with open complement $U$ we have long exact sequence

$$
\rightarrow A^{*-2 c, *^{\prime}-c}(Z) \rightarrow A^{*, *^{\prime}}(X) \rightarrow A^{*, *^{\prime}}(U) \rightarrow A^{*-2 c+1, *^{\prime}+c}(Z) \rightarrow .
$$

Indeed we have the Thom isomorphism (see [10],[8])

$$
A^{*, *^{\prime}}(X / U) \cong A^{*, *^{\prime}}\left(T h_{X}(Z)\right) \cong A B P^{*-2 c, *^{\prime}-c}(Z)
$$

where $T h_{X}(Z)$ is the Thom space of the normal bundle for $Z \subset X$.

From Corollary 2.8 and Corollary 5.3 , we know that $\mathcal{M}_{A B P}(k)$ and $\mathcal{M}_{C H}(k)$ have the same set of isomorphism classes of objects. Hence we also consider the motive $M_{\alpha}^{A B P}$ for $A B P^{2 *, *}$-theory. We have the canonical map: $\tilde{f}$ : $M^{A B P}\left(Q_{\alpha}\right)=\oplus_{i=0}^{2^{n-1}-1} M_{\alpha}^{A B P}(i)[2 i] \rightarrow \oplus_{i=1}^{2^{n-1}-1} M_{\alpha}^{A B P}(i)[2 i]=M^{A B P}\left(Q_{\xi}\right)(1)[2]$. 
We know that $\left.f\right|_{M_{\alpha}(i)[2 i]}$ is an isomorphism. From Lemma 2.1 it follows that $\left.\tilde{f}\right|_{M_{\alpha}^{A B P(i)[2 i]}}$ is an isomorphism too. From the above corollary and the long exact sequence, we have the isomorphism

$$
A B P^{2 *, *}\left(M_{\alpha}^{A B P}\right) \cong A B P^{2 *, *}\left(U_{\alpha}\right) .
$$

Of course $A B P^{*, *^{\prime}}\left(U_{\alpha}\right)$ is $A B P$-theory of a space, so there is AHss converging to $A B P^{*, *}\left(U_{\alpha}\right)$ and the cohomology operations act on $H^{*, *}\left(U_{\alpha} ; \mathbb{Z} / 2\right)$.

Let $\pi: U_{\alpha} \subset Q_{\alpha} \rightarrow C\left(Q_{\alpha}\right)=\chi_{\alpha}$ be the induced map. Then the map

$$
\pi^{*}: H^{, *, *}\left(\chi_{\alpha} ; \mathbb{Z} / 2\right) \rightarrow H^{*, *}\left(U_{\alpha} ; \mathbb{Z} / 2\right) \cong H^{, * *}\left(M_{\alpha} ; \mathbb{Z} / 2\right)
$$

is isomorphic for $[\mathrm{deg}]-(\mathrm{deg})>0$ and $[\mathrm{deg}] \leq 2^{n}-2$ from Corollary 6.2. In particular, we can canonically identify $\alpha^{\prime} \in H^{n, n-1}\left(U_{\alpha} ; \mathbb{Z} / 2\right)$ and $c_{i}=Q_{0} \ldots \hat{Q}_{i} \ldots Q_{n-2}\left(\alpha^{\prime}\right) \in H^{2 * *}\left(U_{\alpha} ; \mathbb{Z} / 2\right)$ as in Corollary 6.2 and Corollary 6.3. From the proof of Corollary 5.2, we recall $F_{1}^{2 *, *}=B P^{<0} F_{0}^{2 *, *}$ for the associated filtration. Hence a lift in $A B P^{2 * *}\left(U_{\alpha}\right)$ of an element in $H^{2 *, *}\left(U_{\alpha} ; \mathbb{Z}_{(2)}\right) \subset \operatorname{gr} A B P^{2 *, *}\left(U_{\alpha}\right)$ is well defined modulo $I(2)$. Let $\bar{c}_{i} \in$ $A B P^{2 *, *}\left(U_{\alpha}\right)$ be lifts of $c_{i}$.

We need the following lemma.

Lemma 6.5 Let $S=\left(x_{i_{1}}, \ldots\right)$ be a sequence from Theorem 5.1. Then for smooth $X$, we have the $M U_{(p)}^{*}$-module isomorphism

$$
A M U(S)^{2 * *}(X) \cong A M U^{2 *, *}(X) /(S)
$$

Proof: Let $S=R \cup\left\{x_{j}\right\}$ and $x_{j} \notin R$. Consider the long exact sequence induced from the cofiber sequence given in Section 5

$$
\begin{aligned}
\rightarrow A M U(R)^{2 *+2 j, *+j}(X) \stackrel{x_{j}}{\rightarrow} A M U(R)^{2 *, *}(X) & \rightarrow A M U(S)^{2 * *}(X) \\
& \stackrel{\delta}{\rightarrow} A M U(R)^{2 *+2 j+1, *+j}(X) \rightarrow .
\end{aligned}
$$

Here $A M U(R)^{2 *+2 j+1, *+j}(X)=0$ from Remark (1),(2) after the Theorem 5.1. By induction, we assume $A M U(R)^{2 * *}(X) \cong A M U^{2 *, *}(X) /(R)$. Then we have

$$
A M U(S)^{2 *, *}(X) \cong A M U(R)^{2 *, *}(X) /\left(x_{j}\right) \cong A M U^{2 *, *}(X) /\left(R, x_{j}\right) .
$$

Thus we get the lemma. 
In particular, the case $A M U(S)=A H \mathbb{Z}_{(p)}$ is Corollary 5.2. Moreover, we use the case $A M U(S)=A k(n)$,

$$
(*) \quad A k(n)^{2 *, *}(X) \cong A B P^{2 *, *}(X) /\left(p, v_{1}, \ldots, \hat{v}_{n}, \ldots\right)
$$

in the lemma bellow.

Lemma 6.6 In $A B P^{2 *, *}\left(U_{\alpha}\right)$ there are the following relations:

$$
v_{i} \bar{c}_{j}+v_{j} \bar{c}_{i}=0 \bmod \left(I(2)^{2}\right), \quad \text { for } 0 \leq i<j \leq n-2 .
$$

Proof: Take $z=Q_{0} \ldots \hat{Q}_{i} \ldots \hat{Q}_{j} \ldots Q_{n-2}\left(\alpha^{\prime}\right)$ in $H^{* * *}\left(U_{\alpha} ; \mathbb{Z} / 2\right)$ so that $w(z)=$ 1 , and $Q_{i}(z)=c_{j}, Q_{j}(z)=c_{i}$ for the construction of $c_{i}$ in Corollary 6.3. We consider AHss for $A k(i)$-theory

$$
E_{2}^{*, *, *}\left(U_{\alpha}\right)=H^{*, *}\left(U_{\alpha} ; k(i)^{*}\right) \Longrightarrow A k(i)^{*, *}\left(U_{\alpha}\right)
$$

The first nonzero differential is given by Lemma 5.5

$$
d_{2^{i+1}-1}(z)=v_{i} \otimes\left(Q_{i}+\sum a_{I J} P^{I} Q_{J}\right)(z)
$$

Here $Q_{J}(z)=0$ follows from $w\left(Q_{J}(z)\right) \leq-1$ since $|J| \geq 2$. Thus we know

$$
\text { (1) } \quad d_{2^{i+1}-1}(z)=v_{i} \otimes Q_{i}(z)=v_{i} \otimes c_{j}
$$

Hence $v_{i} c_{j}=0$ in $\operatorname{gr} A k(i)^{2 *, *}\left(U_{\alpha}\right)$ and this implies

$$
\text { (2) } \quad v_{i} \tilde{c}_{j}=0 \quad \bmod \left(F_{-\left|v_{i}\right|+1}=\left(v_{i}^{2}\right)=I(2)^{2}\right) \quad \text { in } A k(i)^{2 *, *}\left(U_{\alpha}\right)
$$

where $\tilde{c}_{i}$ is a lift of $c_{i}$ into $A k(i)$-theory. Since $\bar{c}_{j}$ is a lift of $c_{j}$ in $A B P^{2 *, *}\left(U_{\alpha}\right)$, from $(*)$, we get:

(3) $v_{i} \bar{c}_{j}=0 \quad \bmod \left(I(2)^{2}, 2, v_{1}, \ldots, \hat{v}_{i}, \ldots\right) \quad$ in $A B P^{2 *, *}\left(U_{\alpha}\right)$.

Note, that $[\operatorname{deg}]\left(v_{i} \bar{c}_{j}\right)=2^{n}-2^{i+1}-2^{j+1}+2$. But, if $[\operatorname{deg}]\left(v_{s} \bar{c}_{t}\right)$ is the above degree, then $(s, t)=(i, j)$ or $(j, i)$.

From Corollary 5.2 and Corollary 6.3 , we see that $A B P^{2 *, *}\left(U_{\alpha}\right)$ is generated by $1, \bar{c}_{0}, \ldots, \bar{c}_{n-2}$ as a $B P^{*}$-module. Then we can write (3) as

$$
\text { (4) } v_{i} \bar{c}_{j}+\lambda_{j} v_{j} \bar{c}_{i}=0 \quad \bmod \left(I(2)^{2}\right), \quad \in A B P^{2 *, *}\left(U_{\alpha}\right)
$$


for some $\lambda_{j} \in \mathbb{Z} / 2$. Considering similar exact sequence for $A k(j)$-theory, we get equation:

$$
\text { (5) } \quad \lambda_{i} v_{i} \bar{c}_{j}+v_{j} \bar{c}_{i}=0 \quad \bmod \left(I(2)^{2}\right) \in A B P^{2 *, *}\left(U_{\alpha}\right),
$$

for some $\lambda_{i} \in \mathbb{Z} / 2$. Now, either (4), or (5), or their sum gives us the desired relation.

Remark. We can prove the following fact $([22],[14])$.

For $y_{m} \in A B P^{*, *}(X), 0 \leq m$, suppose that $\sum_{m} v_{m} y_{m}=0$ in $A B P^{*, *}(X)$. Then there is $x \in H^{*, *}(X ; \mathbb{Z} / p)$ such that $Q_{m}(x)=\rho\left(y_{m}\right) \in H^{*, *}(X ; \mathbb{Z} / p)$ for all $m$ where $\rho: A B P \rightarrow A H \mathbb{Z} / p$ is the natural (Thom) map.

In our case, $\sum v_{m} y_{m}=v_{i} \bar{c}_{j}+v_{j} \bar{c}_{i} \bmod \left(I(2)^{2}\right)$ and $z=x$. The above lemma can be also proven by using the above fact (and (3) in the proof of the lemma), since $z$ with $Q_{i}(z)=c_{j}$ is uniquely determined as $z=Q_{0} \ldots \hat{Q}_{i} \ldots \hat{Q}_{i} \ldots Q_{n-1}\left(\alpha^{\prime}\right)$.

Corollary 6.7 If $i<j$, then $v_{i} \bar{c}_{j}=0 \bmod \left(\bar{c}_{0}, \ldots, \bar{c}_{j-1}\right)$ in $A B P^{2 * *}\left(U_{\alpha}\right)$.

Proof: From above lemma, we get this result $\bmod \left(I(2)^{2}\right)$. For $k>j$, we see,

$$
[\operatorname{deg}]\left(y_{k} \bar{c}_{k}\right) \leqslant[\operatorname{deg}]\left(\bar{c}_{k}\right)=2^{n}-2^{k}<2^{n}-2^{i+1}-2^{j+1}+2=[\operatorname{deg}]\left(v_{i} \bar{c}_{j}\right) .
$$

Hence we have a relation:

$$
\tilde{v}_{i} \bar{c}_{j}=0 \quad \bmod \left(\bar{c}_{0}, \ldots, \bar{c}_{j-1}\right)
$$

where $\tilde{v}_{i}=v_{i} \bmod \left(I(2)^{2}\right)$. To prove that we can assume that $\tilde{v}_{i}=v_{i}$, we have to use the induction on $i$.

From Lemma 5.4, we get the $A M U$-version.

Corollary 6.8 There are relations for $0 \leq i<j \leq n-2$,

$$
v_{i} \bar{c}_{j}+v_{j} \bar{c}_{i}=0 \bmod \left(I(2)^{2}\right) \quad \text { in } M G L^{2 *, *}\left(U_{\alpha}\right) .
$$

Moreover, if $i<j$, then $v_{i} \bar{c}_{j}=0 \bmod \left(\bar{c}_{0}, \ldots, \bar{c}_{j-1}\right)$ in $M G L^{2 *, *}\left(U_{\alpha}\right)$. 


\section{7 injectivity for $M G L^{2 *, *}$}

From Corollary 2.8 and Corollary 5.3, we know that $\mathcal{M}_{M G L}(k)$ and $\mathcal{M}_{C H}(k)$ have the same set of isomorphism classes of objects. Hence we can define $M_{\alpha}^{M G L}$ and consider $M G L^{2 *, *}\left(M_{\alpha}^{M G L}\right) \cong M G L^{2 *, *}\left(U_{\alpha}\right)$, let us write it simply $M G L^{2 *, *}\left(M_{\alpha}\right)$.

Let $\bar{k}$ be the algebraic closure of $k$ and $\left.X\right|_{\bar{k}}=X \otimes_{k} \bar{k}$. Let

$$
a c: M G L^{2 *, *}(X) \rightarrow M G L^{2 *, *}\left(\left.X\right|_{\bar{k}}\right)
$$

be the induced map. Since $\left.M_{\alpha}^{M G L}\right|_{\bar{k}}$ is a direct sum of $(M G L)$ Tate-motives, we have:

$$
M G L^{2 *, *}\left(\left.M_{\alpha}\right|_{\bar{k}}\right) \cong M U^{*} \otimes C H^{*}\left(\left.M_{\alpha}\right|_{\bar{k}}\right) \cong M U^{*}\{1, \bar{c}\}
$$

where $\operatorname{deg}(\bar{c})=\left(2^{n}-2,2^{n-1}-1\right)$. The element $\bar{c}$ here is the image of $e_{0} \in$ $\Omega_{0}\left(M_{\alpha}\right)$ (defined in Section 3) under the natural map $\Omega^{*}(X) \rightarrow M G L^{2 * * *}(X)$.

Recall also that $M G L^{2 *, *}\left(M_{\alpha}\right)$ is generated by $1, \bar{c}_{0}, \ldots, \bar{c}_{n-2}$ as a $M U^{*}$ module (compare with the Theorem 3.4).

Lemma 7.1 We have ac $(1)=1$ and $a c\left(\bar{c}_{i}\right)=v_{i} \bar{c} \bmod (I(2, i-1))$. Hence

$$
a c\left(M G L^{2 * * *}\left(M_{\alpha}\right)\right)=M U^{*}\{1\} \oplus I(2, n-2)\{\bar{c}\} .
$$

Proof: Since for arbitrary $E / k$, the map $C H^{2^{n-1}-1}\left(M_{\alpha}\right) \rightarrow C H^{2^{n-1}-1}\left(R_{\alpha}\right)$ is an isomorphism, and for anisotropic quadric $Q$ of dimension $d$, the map

$$
a c^{C H}: \mathbb{Z} \cong C H^{d}(Q) \rightarrow C H^{d}\left(\left.Q\right|_{\bar{k}}\right) \cong \mathbb{Z}
$$

is the multiplication by 2, we get: $a c^{C H}\left(c_{0}\right)=2 c$, where $c$ is the generator of $C H^{2^{n-1}-1}\left(\left.M_{\alpha}\right|_{\bar{k}}\right) \cong \mathbb{Z}$. Since $\operatorname{deg}\left(M U^{*}\right) \leq 0$, we note that $[\operatorname{deg}]\left(M G L^{2 * * *}\left(M_{\alpha}\right)\right) \leq$ $2 \operatorname{dim}\left(R_{\alpha}\right)$ by Corollary 5.2 . Hence we know

$$
M G L^{2^{n}-2,2^{n-1}-1}\left(M_{\alpha}\right) \cong C H^{2^{n-1}-1}\left(M_{\alpha}\right) \cong \mathbb{Z}
$$

Thus we see that $a c\left(\bar{c}_{0}\right)=2 \bar{c}$ also in $M G L^{2 *, *}\left(\left.M_{\alpha}\right|_{\bar{k}}\right)$.

The relation $v_{i} \bar{c}_{0}=2 \bar{c}_{i} \bmod \left(I(2)^{2}\right)$ in Corollary 6.8 implies at first $a c\left(\bar{c}_{i}\right) \in$ $I(2) \bar{c}$. Since

$$
\left(v_{i} \bar{c}_{0}-2 \bar{c}_{i}\right) \in I(2)^{2}\left\{\bar{c}_{0}, \ldots, \bar{c}_{n-2}\right\}
$$


(note that $[\operatorname{deg}]\left(v_{i} \bar{c}_{0}-2 \bar{c}_{i}\right)>0$ but $\left.[\operatorname{deg}](1)=0\right)$, we have $a c\left(v_{i} \bar{c}_{0}-2 \bar{c}_{i}\right) \in$ $I(2)^{3}\{\bar{c}\}$. Hence we get

$$
a c\left(\bar{c}_{i}\right)=\left(v_{i} / 2\right) a c\left(\bar{c}_{0}\right)=v_{i} \bar{c} \bmod \left(I(2)^{2} \bar{c}\right)
$$

from Corollary 6.8 since $M G L^{2 * * *}\left(\left.M_{\alpha}\right|_{\bar{k}}\right)$ is $M U^{*}$-free.

Compare with Proposition 4.4.

Theorem 7.2 The map ac: $M G L^{2 *, *}\left(M_{\alpha}\right) \rightarrow M G L^{2 *, *}\left(\left.M_{\alpha}\right|_{\bar{k}}\right)$ is injective and

$$
M G L^{2 *, *}\left(M_{\alpha}\right) \cong M U^{*}\{1\} \oplus I(2, n-2)\{\bar{c}\} .
$$

Proof: Consider the filtration

$$
\left(2, \ldots, v_{n-2}\right)=I(2, n-2) \supset I(2, n-3) \supset \ldots \supset I(2,0)=(2) .
$$

Then we have the isomorphism $I(2, i) / I(2, i-1) \cong M U^{*} / I(2, i-1)\left\{v_{i}\right\}$. Thus we get the isomorphism

$$
\operatorname{gr} I(2, n-2)\{\bar{c}\} \cong \oplus_{0 \leq i \leq n-2} M U^{*} / I(2, i-1)\left\{v_{i} \bar{c}\right\} .
$$

Let $A$ be the $M U^{*}$-submodule of $M G L^{2 *, *}\left(M_{\alpha}\right)$ generated by $\left\{\bar{c}_{0}, \ldots, \bar{c}_{n-2}\right\}$, i.e., $M G L^{2 *, *}\left(M_{\alpha}\right) \cong M U^{*}\{1\} \oplus A$. Then from Lemma 7.1, we have the map $g=\left.a c\right|_{A}: A \rightarrow I(2, n-2)\{\bar{c}\}$. Let $A_{i}$ be the submodule of $M G L^{2 *, *}\left(M_{\alpha}\right)$ generated by $\left\{\bar{c}_{0}, \ldots, \bar{c}_{i}\right\}$. Since $a c\left(\bar{c}_{i}\right)=v_{i} \bar{c} \bmod (I(2, i-1))$, we have the induced map

$$
\operatorname{gr}(g): \operatorname{gr} A=\oplus A_{i} / A_{i+1} \rightarrow \oplus_{0 \leq i \leq n-2} M U^{*} / I(2, i-1)\left\{v_{i} \bar{c}\right\} .
$$

Here from Corollary 6.8 , the module $A_{i} / A_{i-1}$ is a quotient of the module

$$
M U^{*}\left\{\bar{c}_{0}, \ldots, \bar{c}_{i}\right\} /\left(v_{k} \bar{c}_{i}-v_{i} \bar{c}_{k}, \bar{c}_{0}, \ldots, \bar{c}_{i-1} \mid k<i\right) \cong M U^{*} / I(2, i-1)\left\{\bar{c}_{i}\right\} .
$$

Here note $\operatorname{gr}(g)\left(A_{i} / A_{i-1}\right)=M U^{*} / I(2, i-1)\left\{v_{i} \bar{c}\right\}$. Hence $\left.\operatorname{gr}(g)\right|_{\left(A_{i} / A_{i-1}\right)}$ is isomorphic and so are $\operatorname{gr}(g)$ and $g$.

Corollary 7.3 There are $B P^{*}$-module isomorphisms

$$
\begin{aligned}
& \operatorname{gr} A B P^{2 *, *}\left(U_{\alpha}\right) \cong\left(B P^{*}\{1\} \oplus \oplus_{i=0}^{n-2} B P^{*} / I(2, i-1)\left\{c_{i}\right\}\right), \\
& A B P^{2 *, *}\left(U_{\alpha}\right) \cong\left(B P^{*}\left\{1, \bar{c}_{0}, \ldots, \bar{c}_{n-2}\right\} /\left(v_{i} \bar{c}_{j}-v_{j} \bar{c}_{i} \mid i<j\right)\right) .
\end{aligned}
$$


Note that $A B P^{2 *, *}\left(Q_{\alpha}\right)$ is a free $A B P^{2 *, *}\left(U_{\alpha}\right)$-module

$$
A B P^{2 * * *}\left(Q_{\alpha}\right) \cong A B P^{2 *, *}\left(U_{\alpha}\right)\left\{1, h, \ldots, h^{2^{n-1}-1}\right\}
$$

where $\operatorname{deg}(h)=(2,1)$. Similar fact also holds for $\operatorname{gr} A B P^{2 * *}\left(Q_{\alpha}\right)$. However note that $h^{2^{n-1}} \neq 0 \in A B P^{2 *, *}\left(Q_{\alpha}\right)$.

Let $\pi: X \rightarrow p t$ is the projection map and $I(X)=\pi_{*} M G L^{2 *, *}(X)$. Let $I^{\prime}(X)$ be the sub $M U^{*}$-module of $I(X)$ generated by $\pi_{*}$-images of elements of $M G L^{2 *, *}(X)$ of positive degree.

Theorem 7.4 We have $\pi_{*}(1)=v_{n}$ and $\pi_{*}\left(\bar{c}_{i}\right)=v_{i}$. Hence $I^{\prime}\left(M_{\alpha}^{M G L}\right) \cong$ $I(2, n-2)$ and $I\left(M_{\alpha}^{M G L}\right) \cong I(2, n-1)$.

Proof: Since $t_{\mathbb{C}}\left(P_{\alpha}\right)=v_{n-1}$, we have $\pi_{*}(1)=v_{n-1}$. For $\pi_{*}\left(\bar{c}_{0}\right)$, we know $\pi_{*}\left(c_{0}\right)=\operatorname{deg}\left(P_{\alpha}\right)=2$ in $C H^{0}(p t.) \cong \mathbb{Z}$. Hence $\pi_{*}\left(\bar{c}_{i}\right)=v_{i}$ follows from the relation $2 \bar{c}_{i}=v_{i} \bar{c}_{0}$ in $M G L^{2 *, *}\left(M_{\alpha}\right)$.

\section{References}

[1] V.Chernousov, S.Gille, A.Merkurjev, Motivic decomposition of isotropic projective homogeneous varieties, Preprint, SFB 478 - Geometrische Strukturen in der Mathematik des Mathematischen Instituts der Westfälischen Wilhelms-Universität Münster, 264, May 2003. (wwwmath.uni-muenster.de/math/inst/sfb/about/publ/heft264.ps)

[2] D.Hoffmann. Isotropy of quadric forms over the function field of a quadric, Math. Z. 220 (1995), 461-476.

[3] P.Landweber. Annihilater ideals and complex bordism, Illinois J. Math. 17 (1973), 273-284.

[4] M.Levine, F.Morel, Algebraic cobordism I, Preprint, 1-116.

[5] M.Levine, F.Morel, Cobordisme algébrique I, C.R.Acad. Sci. Paris, Série I, Math 332 (2001) no. 8, 723-728.

[6] M.Levine, F.Morel, Cobordisme algébrique II, C.R.Acad. Sci. Paris, Série I, 332 (2001), 815-820. 
[7] A.Merkurjev, Rost degree formula, Preprint, 2000, 1-19.

[8] A.Nenashev, K.Zainoulline, On Oriented Cohomology for Relative Cellular Spaces, Preprint, May 2004.

[9] D.Orlov, A.Vishik, V.Voevodsky. An exact sequence for Milnor's $K$ theory with applications to quadric forms. www.math.uiuc.edu/Ktheory/0454 (2000).

[10] I.Panin, Oriented Cohomology Theories of Algebraic Varieties, K-theory J. 30 (2003), 265-314.

[11] M.Rost, Some new results on the Chow groups of quadrics, Preprint, Regensburg, 1990.

[12] M.Rost, The motive of a Pfister form, Preprint, 1998.

[13] D. Quillen. Elementary proofs of some results of cobordism theory using Steenrod operations. Adv. Math. 7 (1971), 29-56.

[14] H.Tamanoi. Spectra of BP-linear relations, $v_{n}$-series, and BP cohomology of Eilenberg-MacLane spaces. Trans. Amer. Math. Soc. 352 (2000), 5139-5178.

[15] A.Vishik, Symmetric operations (in Russian), Trudy Mat. Ins. Steklova 246 (2004), Algebr. Geom. Metody, Svyazi i Prilozh., 92-105.

[16] A.Vishik, Symmetric operations II, in preparation.

[17] V.Voevodsky The Milnor conjecture www.math.uiuc.edu/K-theory/0631 (1996).

[18] V.Voevodsky, Triangulated categories of motives over a field, in Cycles, Transfers and motivic homology theories (by E.M.Friedlander, A.Suslin and V.Voevodsky), Princeton University Press, 2000.

[19] V.Voevodsky, Motivic Cohomology with $\mathbb{Z} / 2$-coefficients, Publ. Math. Inst. Hautes Études Sci. 98 (2003), 59-104.

[20] V.Voevodsky. Reduced power operations in motivic cohomology. Publ.Math. IHES 98 (2003),1-57. 
[21] V.Voevodsky. On motivic cohomology with $\mathbb{Z} / l$-coefficients. www.math.uiuc.edu/K-theory/0631 (2003).

[22] N. Yagita. On relations between Brown-Peterson cohomology and the ordinary mod $p$ cohomology theory. Kodai Math.J 7 (1984), 273-285.

[23] N. Yagita. Applications of Atiyah-Hirzebruch spectral sequence for motivic cobordism. Proc. London Math. Soc. 90 (2005), 783-816. 